\title{
Myelodysplastic syndrome with ring sideroblasts, SF3B1 mutation and beta thalassemia trait: a case of report
}

\author{
Amin Hojat ${ }^{1}$, Grace Kao ${ }^{2}$, Monika Pilichowska ${ }^{1 *}$ \\ ${ }^{1}$ Department of Pathology and Laboratory Medicine, Tufts Medical Center, Boston MA 02111, USA; \\ ${ }^{2}$ MiltenyiBiotec, Inc. Somerville, MA 02143, USA
}

Keywords: myelodysplastic syndrome, anemia, beta thalassemia, ring sideroblast, SF3B1

\section{INTRODUCTION}

Anemia is the most frequent blood disorder and is defined as a decrease in red blood cell (RBC) mass. Hemoglobin $<135 \mathrm{~g} / \mathrm{L}$ or hematocrit $(\mathrm{HCT})<0.41 \mathrm{rep}-$ resents anemia in men while the values for women are $<120 \mathrm{~g} / \mathrm{L}$ or $<0.36$ respectively ${ }^{[1]}$. Globally, anemia affects 1.62 billion people(24.8\% of the population). World Health Organization regional estimates generated for preschool-age children and pregnant and nonpregnant women indicated that the highest incidence was in South-East Asia, where 315 million individuals in these three population groups were affected, with highest prevalence reported in preschool-age children ${ }^{[2]}$. Anemia is a symptom that requires investigation to determine its underlying etiology. This can be difficult as multiple causes need to be considered, falling into three independent mechanisms: decreased $\mathrm{RBC}$ production (ineffective erythropoiesis), increased RBC destruction and blood loss. Thalassemia, sideroblastic anemias and myelodysplastic syndromes are common causes of ineffective erythropoiesis. Thalassemia is a group of hereditary blood disorders, characterized by abnormal hemoglobin production as a result of mutations in either alpha or beta-globin chain genes of hemoglobin. Defects involving the incorporation of iron into the heme molecule result in sideroblastic anemias. Sideroblastic anemias may be either congenital or acquired. The most commonly acquired sideroblastic anemia is myelodysplastic syndrome with ring sideroblasts(MDS-RS). It has been shown that the splicing factor $3 \mathrm{~b}$ subunit 1 gene (SF3B1) is frequently mutated in MDS-RS ${ }^{[3]}$. Concurrent cases of MDS-RS and beta thalassemia are rare and can present adiagnostic challenge.

\section{CASE REPORT}

A 60-year-old man of Chinese ethnicity presented to the hospital for evaluation of anemia. He had a longstanding history of normocytic anemia (hemoglobin range of 75-91 g/L), diabetes and elevated ferritin $(>2247 \mathrm{pmol} / \mathrm{L})$. He did not require blood transfusions prior to presentation. His family history included mother and father with hypertension and otherwise no other blood disorders or anemia. On presentation, laboratory results showed moderate to severe anemia (hemoglobin $78 \mathrm{~g} / \mathrm{L}$, HCT 0.24), normal total bilirubin $(17.1 \mu \mathrm{mol} / \mathrm{L})$, normal reticulocyte count $(1.7 \%)$ and elevated lactate dehydrogenase (LDH 279 U/L). Other parts of his complete blood count (CBC) including white blood cell count $\left(8.2 \times 10^{9} / \mathrm{L}\right)$, platelets $\left(304 \times 10^{9} / \mathrm{L}\right)$ and mean corpuscular volume(MCV 87 $\mathrm{fL}$ ) were normal. Examination of hisperipheral blood smear showed abnormal red blood cell morphology

*Correspondence to: Monika Pilichowska, MD, Department of Pathology and Laboratory Medicine, Tufts Medical Center, 800 Washington Street, Boston, MA 02111, USA. Tel: 1-617-636-1112;Fax: 1-617-636-7128; E-mail: mpilichowska@tuftsmedicalcenter.org The authors declared no conflict of interests. 
including target cells, tear drop cells as well as schistocytes and basophilic stippling(Fig.1 A ). Hemoglobin electrophoresis identified an A/F pattern with $10.4 \%$ hemoglobin F and 2.8\% hemoglobin A2. Molecular analysis by targeted sequencing of the alpha and betaglobin genes revealed that the patient was heterozygous for IVS II $-654 \mathrm{C}>\mathrm{T} \beta+/$ thalassemia. Because the findings were unusual (hemoglobin A2 level was below diagnostic threshold for beta thalassemia and hemoglobin F level was elevated), further workup was pursued. The bone marrow biopsy was performed to reveal increased cellularity(80\%-90\%), erythroid hyperplasia as well as erythroid dyspoiesis (Fig.1B,C). In addition, numerous ring sideroblasts $(>90 \%$; Fig.1D) were identified on Prussian blue iron stain. Myeloid maturation was complete without significant dyspoiesis. Megakaryocytes were adequate. Cytogenetic stud- ies identified loss of chromosome $\mathrm{Y}$, but otherwise normal chromosome complement 45 (X) (Fig.2). Next Generation Sequencing analysis detected a hot spot missense mutation in SF3B1(p.Lys700 Glu). Based on the clinical and laboratory data, a diagnosis of myelodysplastic syndrome with ring sideroblasts was made in addition to the diagnosis of beta thalassemia trait. Abdominal magnetic resonance imaging(MRI) was performed to determine the extent of tissue iron deposits in the liver and revealed anestimated hepatic iron concentration (HIC) of $2.30-3.50(\mathrm{mg} / \mathrm{g}$ dry weight) which was confirmatory for secondary hemochromatosis. At present, 11 months after initial diagnosis, the patient is doing well although he requires transfusion support with two units of packed red blood cells every 2-3 weeks and iron chelation with deferasirox (Jadenu), $360 \mathrm{mg}$, oral twice a day.

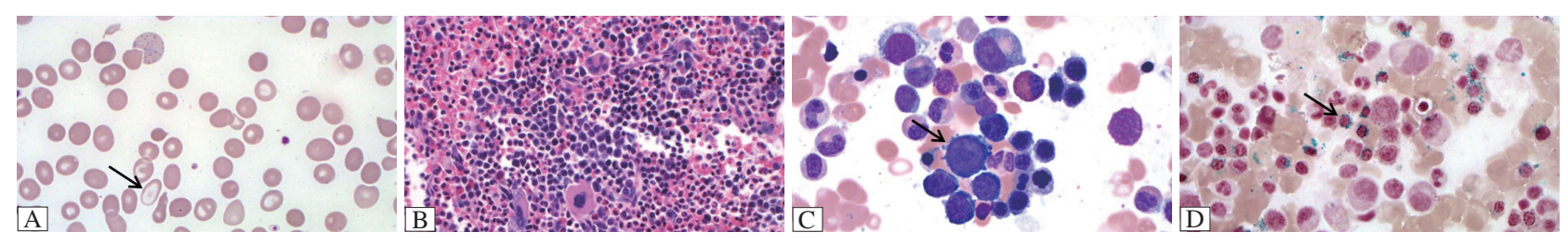

Fig. 1 The images of peripheral blood smear and bone marrow of the patient. A: Peripheral blood smear with anisopoikilocytosis, target cells (arrow), tear drop cells, microspherocytes, basophilic stippling as well as schistocytes (Wright Giemsa stain, $\times 1000$ ); B: Bone marrow core biopsy with trilineage maturing hematopoiesis in a hypercellular marrow as well as erythroid hyperplasia and dyspoiesis( HE stain, $\times 400$ ); C. Bone marrow aspirate with erythroid hyperplasia, dyspoiesis, erythroid precursors with megaloblastoid changes and irregular nuclear contour (arrow)(Wright Giemsa stain, $\times 1000$ ); D. Bone marrow aspirate with ring sideroblasts (arrow)(Prussian blue iron stain, $\times 1000)$.

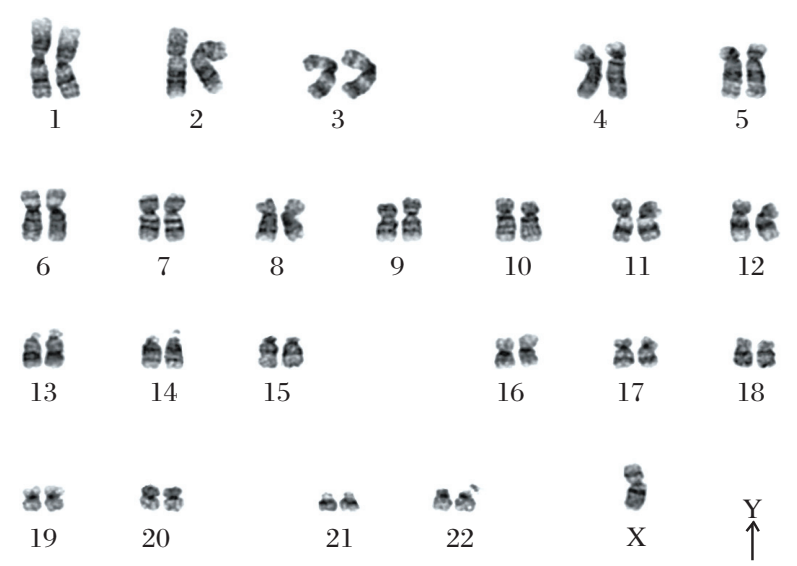

Fig.2 Cytogenetic analysis by conventional karyotyping revealed loss of chromosome Y (arrow) but no other abnormalities.

\section{DISCUSSION}

Myelodysplastic syndrome with ring sideroblasts (MDS-RS), formerly known as refractory anemia with ring sideroblasts (RARS), is characterized by anemia with erythroid dysplasia and ring sideroblasts (RS). By definition, RS should constitute $\geqslant 15 \%$ of the bone marrow erythroid precursors. MDS-RS is usually diagnosed in older individuals and accounts for $3 \%-11 \%$ of all MDS cases. SF3B1 gene mutation on chromosome 2 occurs in the majority of cases with the MDS-RS phenotype (80\%-90\% of MDS-RS-SLD) ${ }^{[3]}$. SF3B1 gene encodes a core component of the U2 small nuclear ribonucleoproteins (snRNP) spliceosome that is critical for ribonucleic acid (RNA) splicing. Haploinsufficiency of SF3Bl is associated with analtered and distinct gene expression profile, including altered splicing of mitochondrial iron transporter gene $\mathrm{ABCB} 7$ and other mitochondrial metabolism genes ${ }^{[4]}$. These genetic changes may lead to ineffective erythropoiesis and ring sideroblasts. In the presence of SF3B 1 mutation, the diagnosis of MDS-RS can be made with less than $5 \%$ marrow ring sideroblasts. MDS-RS with single lineage dysplasia (MDS-RS SLD) with SF3B1 mutation has been associated with overall good prognosis (median overall survival of 69 to 108 months) and a lower risk of leukemic transformation $(1 \%-2 \%)^{[3]}$. It is important to note that ring sideroblasts can be seen in conditions other than MDS, including copper deficiency, vitamin B6 deficiency, lead poisoning and excessive alcohol 
consumption.

Beta thalassemias are characterized by mutations of the beta-globin gene leading to reduced hemoglobin synthesis. Depending on the degree of quantitative reduction in the output of the beta chains, they are classified as $\beta 0 / \beta+/ \beta++$ thalassemia. Beta thalassemia results from over 200 different point mutations and deletions in the beta globin gene (as frequently seen with alpha thalassemia) are rare. Among the most frequent genetic alterations are point mutations of intron sequences resulting in aberrant splicing. The IVS2$654(\mathrm{C}>\mathrm{T})$ point mutation, identified in thisindex case, is a genetic alteration causing beta thalassemia, frequently found in Southeast Asian populations. This creates an aberrant $5^{\prime}$ splice site and activates a cryptic 3 ' splice site within intron 2 of the pre-mRNA (messenger RNA), leading to the retention of $73 \mathrm{nu}-$ cleotides of the second intron ${ }^{[5]}$. In the heterozygous state, or beta thalassemia trait, the clinical symptoms can vary from asymptomatic to mild to moderate microcytic anemia ${ }^{[6]}$.

Both beta thalassemia trait and MDS-RS can present as anemia and show a hypercellular marrow with erythroid hyperplasia in the bone marrow biopsy. MDS-RS usually has morphologic dysplasia in erythroid lineage and may be accompanied by myeloid and megakaryocytic dysplasia in some cases ${ }^{[6]}$. The index case presented with moderate normocytic anemia and had comprehensive bone marrow analysis, including cytogenetic and molecular studies as well as hemoglobin analysis under taken,which revealed a compound heterozygote status for beta thalassemia and SF3B1 mutation as theunderlying causes of his anemia. From diagnostic standpoint, beta thalassemia usually results in increased hemoglobin A2. The index case had low hemoglobin A2, secondary to ineffective erythropoiesis from MDS. In addition, beta thalassemia usually presents as microcytic anemia and MDS-RS presents as normocytic or macrocytic anemia, which would explain normocytic anemia in the index case despite the beta thalassemia trait.

The standard care for patients with myelodysplastic syndrome and decreasing blood counts is constantly changing. Supportive therapy (transfusions, treatment of infections) and hypomethylating agents (such as Decitabine and Azacitidine) are the main components of care to be used with or without Lenalinomide, an immunomodulating agent. Bone marrow transplant is currently the only one curative treatment. Treatment decisions are based on the revised International Prognostic Scoring System (IPSS-R) score including patient's age, comorbidities and the patient's expectations and personal goals. Recently, two separate phase III trials, MEDALIST and BELIEVE, were presented at the 2018 Annual Meeting of the American Society of Hematology (ASH). In these trials, Luspatercept, which is an erythroid maturation agent enhancing latestage erythropoiesis, was found to be safe and effective at reducing transfusion burdens in patients with MDS and beta thalassemia ${ }^{[7]}$. This report emphasized the importance of complete workup in patients with unusual presentations of anemia. Coincidental MDS and/or MDS-RS and beta thalassemia could be more frequent, especially in certain ethnic populations so a high level of suspicion is warranted. Accurate diagnosis is mandated by the availability of novel potentially effective therapies as well as by the need for prognostication and family planning.

\section{References}

[1] The Role of Red Blood Cells in Anemia[EB/OL]. [201908-01]. http://www.hematology.org/Patients/Anemia/

[2] World Health Organization. Worldwide prevalence of anaemia 1993-2005[R]. [2019-08-01]. http://apps.who. int/iris/bitstream/handle/10665/43894/978924159665__ eng.pdf?sequenc $\mathrm{e}=1$,

[3] Swerdlow SH, Campo E, Harris NL, et als. WHO classification of tumours of haematopoietic and lymphoid tissues, revised edition[ M]. IARC:Lyon, France, 2017

[4] Patnaik M, Lasho TL, Hodnefield J, et al. SF3B1 mutations are prevalent in myelodysplastic syndromes with ring sideroblasts but do not hold Independent prognostic value[J]. Blood, 2012,119(2):569-572

[5] Peng X, Ying T, Liu XZ, et al. Both TALENs and CRISPR/Cas9 directly target the HBB IVS2-654 (C > T) mutation in $\beta$-thalassemia-derived iPSCs $[\mathrm{J}]$. Sci Rep, 2015,5:12065.

[6] Jeffrey A, Yanhua W, Yanan F. A rare case of myelodysplastic syndrome with ring sideroblasts, SF3B1 and TET2 mutations in a patient with beta thalassemia trait[J]. North Am J Med Sci, 2017,10(1): 32-35

[7] Fenaux P, Kiladjian JJ, Platzbecker U. Luspatercept for the treatment of anemia in myelodysplastic syndromes and primary myelofibrosis[J]. Blood, 2019, 133:790794.

(Received 24 July 2019, Revised 19 September 2019, Accepted 23 September 2019) 